\title{
Electrochemistry for neurochemical analysis
}

\author{
Ashley E. Ross ${ }^{1}$ (D) $\cdot$ Alexander G. Zestos ${ }^{2}$
}

Accepted: 22 September 2021 / Published online: 12 October 2021

(C) Springer-Verlag GmbH Germany, part of Springer Nature 2021

Over the last several decades, the field of electrochemistry has contributed greatly to the detection of biological molecules in the brain. Early electrophysiology work, dating back as early as the 1600 s, used electrical stimulation to activate nerves, and it is this work that would eventually inspire the incorporation of electrochemical techniques to monitor specific molecules in the brain. Prof. Ralph N. Adams has been generally credited with being the first to implant a carbon-fiber microelectrode in the rat brain to measure catecholamine release. His early work has laid the foundation for in vivo electrochemistry and has inspired the electroanalytical community to further develop electrochemical methods and sensors to probe the complexity of neuron communication.

The detection of neurochemicals is important for understanding basic brain signaling and function. Novel developments in the area of electrochemical detection of neurochemicals have significantly impacted our understanding of basic brain function and important chemical disruptions that occur during neurological disease, addiction, neurodegeneration, and inflammation, and even of the effects of chemotherapy on the brain. The most widely studied neurochemical species in the brain includes the catecholamines dopamine and norepinephrine and their metabolites. Expansion to several electroactive neurochemicals including serotonin, adenosine, histamine, $\mathrm{H}_{2} \mathrm{O}_{2}, \mathrm{NO}, \mathrm{O}_{2}$, ascorbic acid, neuropeptides, and, more recently, guanosine has made huge impacts on our understanding of the dynamics, mechanism, and function of

Published in the topical collection Electrochemistry for Neurochemical Analysis with guest editors Ashley E. Ross and Alexander G. Zestos.

Ashley E. Ross

Ashley.ross@uc.edu

Alexander G. Zestos

zestos@american.edu

1 Department of Chemistry, University of Cincinnati, 312 College Dr., 404 Crosley Tower, Cincinnati, OH 45221-0172, USA

2 Department of Chemistry, American University, 4400 Massachusetts Ave. NW, 218 Hall of Science, Washington, D.C. 20016, USA basic brain signaling. Likewise, electrochemical sensors incorporating enzymes, aptamers, and field effect transistors, to name a few, have enabled expansion to glucose, lactate, glutamate, and choline sensing in the brain. In addition to expanding the analyte toolbox, electrochemical approaches to improve the spatial and temporal resolution of measurement in tissue, new sensors to minimize tissue damage and inflammation, and microelectrode arrays to multiplex tissue detection have enabled exciting advances in neurochemical analysis. All these improvements in electrochemical detection have significantly widened the impact of electrochemistry in neuroscience.

Highly sophisticated electroanalytical methods are necessary for the detection of neurochemicals in complex biological samples. The brain can signal on varying temporal and spatial regimes, and signaling can be impacted based on the brain region of interest, condition of the brain, age, and sex of the organism. When developing electrochemical methods to measure signaling in the brain, it is important to consider this complexity. Novel electroanalytical approaches to improve detection sensitivity, selectivity, temporal and spatial resolution, and multiplexing capabilities have pushed the boundaries of brain detection.

This topical collection highlights exciting new developments in electrochemistry to improve neurochemical detection. This collection spans new electrode materials, microelectrode arrays, improved electrode treatment strategies, fundamental advances in understanding exquisite biosensor design, the electrochemical characterization of novel analytes, and the development of new voltammetric techniques such as rapid pulse voltammetry. Likewise, this collection highlights the application of electrochemical detection to study neurochemicals in single cells, ex vivo, and in vivo, and even new developments to improve chronic in vivo monitoring such as the use of biocompatible reference electrodes.

The future of neurochemical analysis is heavily reliant on further developments in electrochemical sensing. Over the next several years, we anticipate further improvements in electrode biocompatibility, multiplexing capabilities, and 
expansion to novel neurochemical analytes. Further integration of electrochemistry with imaging techniques, microfluidics, and other powerful analytical methods will further push the field of neurochemical sensing. With these future advancements, we anticipate widespread improvements in our understanding of neurochemical signaling cascades in the brain during both health and disease.

We would like to thank the Analytical and Bioanalytical Chemistry editorial team for supporting this topical and timely collection. This past year has been extremely difficult due to the COVID-19 pandemic, and we would like to acknowledge their dedication and passion for continuing to disseminate our science. We would also like to thank all the authors who contributed to this collection from all over the world, despite the ongoing ripples of the pandemic which have inevitably impacted all of our lives and research agendas. Lastly, we would like to thank the reviewers for their dedication to serve the scientific community with their knowledge and expertise.

Publisher's note Springer Nature remains neutral with regard to jurisdictional claims in published maps and institutional affiliations.

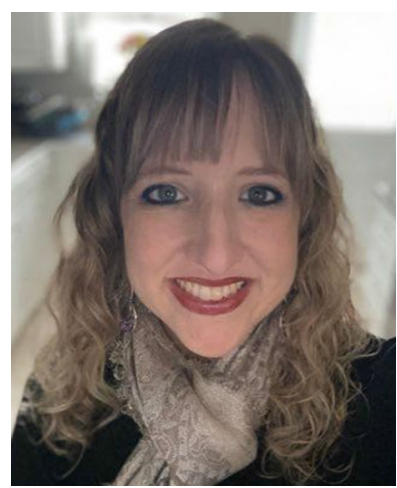

Ashley E. Ross is Assistant Professor of Chemistry and a faculty member of the Neuroscience Research Program at the University of Cincinnati. Her research interests span from investigating the electrode-analyte interface to developing both electrochemical and microfluidic methods to probe complex neurochemical signaling in the brain and immune system. She has been named RCSA Scialog: Microbiome, Neurobiology, and Disease Fellow; Analyst Emerging Investigator; and ChemComm Emerging Investigator, and her work has been highlighted in several themed collections including the ABC's Female Role Models in Analytical Chemistry issue. She serves on the Advisory Board for the Features Panel of ACS Analytical Chemistry and is on the Board of Directors for the Society of Electroanalytical Chemistry.

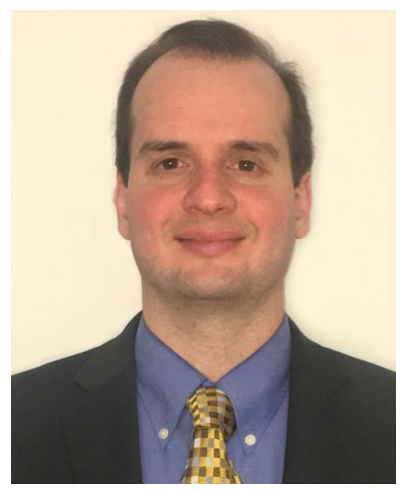

Alexander G. Zestos is Assistant Professor of Chemistry in the Center for Neuroscience and Behavior, American University. His research interests include electrochemical and chromatographic methods for neurotransmitter detection. He investigates the use of novel carbon nanomaterials for biomolecule measurement for many different applications such as studying drugs of abuse, HIV, and others. He has been named Emerging Investigator by the Royal Society

of Chemistry and a finalist for the Csaba Horvath Young Investigator Award, and serves as the editor in chief of The Chemist published by the American Institute of Chemists. He also has served as a manager for the Chemical Society of Washington and mentor for the ACS Project SEED. 